\section{Multiple locations on chromosome 3 are the targets of specific deletions in uveal melanoma}

\begin{abstract}
Purpose Loss of chromosome 3 is a frequent event in uveal melanomas, which is associated with hepatic metastases and a poor prognosis. The entire copy of chromosome 3 is usually lost (monosomy 3); however, a small subset of tumours demonstrate partial deletions of chromosome 3. Analysis of these tumours may allow the identification of tumour suppressor genes (TSGs) that are the molecular target of monosomy 3. Therefore, the purpose of this investigation was to determine the location of these partial deletions of chromosome 3 in uveal melanomas.
\end{abstract}

Methods Microsatellite analysis and restriction fragment-length polymorphism

${ }^{1}$ Academic Unit of Ophthalmology and Orthoptics, Division of Clinical Sciences (South), University of Sheffield, UK

${ }^{2}$ Institute for Cancer Studies, University of Sheffield Medical School, UK

Correspondence: Dr NA Cross, Division of Clinical Sciences (South), Royal Hallamshire Hospital (K-Floor), University of Sheffield, Glossop Road, Sheffield S10 2RX, UK Tel: + 44 (0)114 2712375; Fax: + $44(0) 1142712268$ E-mail: n.a.cross@ shef.ac.uk

Received: 29 July 2004 Accepted: 9 March 2005 Published online: 27 May 2005

This work was funded by Yorkshire Cancer Research
NA Cross ${ }^{1,2}$, A Ganesh², M Parpia², AK Murray², IG Rennie ${ }^{1}$ and K Sisley ${ }^{1}$

Keywords: monosomy 3; deletion; uveal melanoma

\section{Introduction}

Posterior uveal melanomas occur in the choroid and ciliary body of the eye, and account for $80 \%$ of all noncutaneous melanomas. ${ }^{1}$ They are the most common intraocular tumours of adults, having an annual incidence in the western world of 0.8 cases per 100000 population, ${ }^{2}$ and are highly aggressive, frequently metastasising to the liver. Once hepatic metastases are established, survival is usually less than 12 months. ${ }^{3}$ Conversely, iris melanomas, although less frequent, have a good prognosis and only rarely metastasise. ${ }^{4}$

Uveal melanomas have been well characterised by cytogenetic studies, owing largely to their relatively simple karyotypes and ease of culture. Cytogenetic studies on posterior uveal melanoma have revealed the presence of recurrent primary aberrations, including loss of chromosome 3 and gain of chromosome $8 \mathrm{q} \cdot{ }^{5-7}$ Monosomy 3 is present in approximately $50 \%$ of tumours and is associated with a poor prognosis. ${ }^{8-10}$ Postoperative observation of cases with monosomy 3 showed that $57 \%$ of cases developed metastasis within 3 years. ${ }^{11}$ In contrast, tumours with disomy 3 rarely metastasise. ${ }^{11}$ Confirmation of monosomy 3 as a highly sensitive prognostic indicator is supported by other studies, in which its association with a gain of chromosome $8 \mathrm{q}$ confers a poor prognosis. ${ }^{9}$

The recurrent loss of chromosomal regions in tumours indicates the possible location of a tumour suppressor gene (TSG), which can be identified by the detailed mapping of deleted regions. ${ }^{12}$ Deletions of chromosome 3 are frequently observed in many tumour types, although such abnormalities tend to affect only 
chromosome $3 p$, with $3 q$ deletions being a relatively rare event. ${ }^{13}$ Uveal melanomas, however, are highly unusual in that the entire chromosome is subject to loss, and it has been hypothesised that these observations reflect the involvement of one or more TSGs on each chromosomal arm. ${ }^{10,11,14}$ A minority of uveal melanomas do however demonstrate translocations and partial deletions of chromosome 3, occasionally as the sole abnormality. ${ }^{10,15-18}$ Recently, deletion-mapping studies have identified two commonly affected regions at 3p25 and at 3q24-26. ${ }^{18,19}$ To identify additional preferentially lost regions in uveal melanoma, we analysed polymorphic markers along chromosome 3 in a series of 52 primary uveal melanomas, irrespective of chromosome 3 status. Here we describe two possible regions of common loss at 3p25, and a new region at 3p11-14, consistent with previous molecular genetic and cytogenetic findings.

\section{Materials and methods}

\section{Cases}

Prior to collection of blood and tumour samples, local ethical committee approval and informed patient consent were obtained. The research adhered to the tenets of the Declaration of Helsinki. The tumours of 52 patients, treated at the Department of Ophthalmology and Orthoptics, University of Sheffield, between 1994 and 1999 were studied. All were primary uveal melanomas, with no prior treatment and were either locally resected or enucleation. For each case, paired peripheral blood and tumour tissue were available for DNA extraction. The series comprised two anterior uveal melanomas (iris melanomas) and 50 posterior uveal melanomas, including 21 ciliary body tumours, 23 choroidal tumours, and six chorio-ciliary body tumours. The majority of tumours were large tumours ( $>10 \mathrm{~mm}$ diameter) $(n=45)$, only one tumour was medium sized $(5-10 \mathrm{~mm}$ diameter) and the sizes of a further seven tumours, including one iris melanoma, were not recorded.

\section{PCR amplification}

Genomic DNA was isolated from fresh-frozen tumour tissue $(25 \mathrm{mg})$ and from paired patient whole blood $(1 \mathrm{ml})$ using the Mini Genomic DNA extraction kit (Qiagen, Crawley, UK). Restriction fragment-length polymorphism (RFLP) and microsatellite markers were analysed by PCR amplification. A total of 19 polymorphic loci were studied. Primers for microsatellite markers were labelled with either IRD 700 or IRD 800 (MWG-Biotech, Ebersberg, Germany). Microsatellite PCR products were loaded onto $6 \%$ denaturing polyacrylamide gels and run on a Licor dual laser DNA sequencer. $\mathrm{LOH}$ was defined as a visible reduction in the intensity of one allele in the tumour sample, compared to the paired blood sample, and was scored by two independent observers (NAC, AG). In case of disagreement, the result was declared noninformative.

\section{Cytogenetic analysis and fluorescence in situ hybridisation (FISH)}

Uveal melanoma cells were cultured from fresh primary tumour material and cytogenetic analysis was performed as detailed previously. ${ }^{9}$ The description of cytogenetic abnormalities seen in this study was reported using the International System of Human Cytogenetic

Nomenclature (ISCN, 1995). To identify the abnormalities detected by molecular genetic findings, FISH was performed using chromosome 3-specific paints as outlined previously. ${ }^{9}$ Slides were analysed on an Olympus BX50 microscope linked to a CCD camera, and analysed using MacProbe software (PSI Inc.).

\section{Results}

In this study, a panel of uveal melanomas was investigated for the presence of $\mathrm{LOH}$ at 19 polymorphic loci along both arms of chromosome 3 . Of 52 tumours studied, 30 (58\%) demonstrated LOH at all loci tested, which was presumed to represent functional monosomy 3 , and 17 tumours (33\%) demonstrated retention of heterozygosity at all loci (disomy 3). A further five tumours (10\%) showed evidence of structural abnormalities of chromosome 3, with LOH affecting one or more markers, but retention of heterozygosity at other sites. A summary of the five cases demonstrating structural abnormalities of chromosome 3 is shown in Table 1, and patient details of these cases are shown in Table 2 .

Two tumours demonstrated evidence of breakpoints affecting 3p24-26 by microsatellite analysis (Figure 1). SOM 147 demonstrated LOH at all informative loci, except for D3S3525 (3p26). Conversely, SOM 240 demonstrated LOH at D3S3525 (3p26), whereas retention of heterozygosity was observed at all other loci on chromosome $3 \mathrm{p}$. This indicates a possible region of common loss between D3S3525 (3p26) and D3S1293 (3p24) in these two tumours. In addition to the deletion at 3p26, SOM 240 also demonstrated LOH affecting 3q13ter by microsatellite analysis, suggestive of a large deletion, potentially involving the entire $3 q$ arm (Table 1). Subsequent FISH and cytogenetic analysis of SOM 240 indicated the presence of a large deletion affecting $3 q$, but appeared to suggest the presence of a translocation telomeric to the region identified by molecular genetic studies (Figure 2). Such differences could arise either by 
Table 1 Summary of cases of uveal melanoma with structural abnormalities of chromosome 3

\begin{tabular}{|c|c|c|c|c|c|c|c|}
\hline Marker & Band & 177 & 147 & 240 & 169 & 244 & \multirow[b]{2}{*}{ CDR1 } \\
\hline D3S3525 & $3 p 26$ & & & & & & \\
\hline D3S1293 & $3 p 24$ & & & & & & \\
\hline D3S1298 & $3 p 23$ & & & & & & \multirow{14}{*}{ CDR2 } \\
\hline D3S2 & $3 \mathrm{p} 21.3$ & & & & & & \\
\hline D3S32 & $3 p 21.3$ & & & & & & \\
\hline D3F15S2 & $3 p 21.3$ & & & & & & \\
\hline D3S1478 & $3 \mathrm{p} 21.3$ & & & & & & \\
\hline D3S1573 & $3 \mathrm{p} 21.2$ & & & & & & \\
\hline D3S1481 & $3 p 14.2$ & & & & & & \\
\hline D3S30 & $3 \mathrm{p} 12$ & & & & & & \\
\hline D3S4529 & $3 \mathrm{p} 11$ & & & & & & \\
\hline D3S1251 & $3 q 11$ & & & & & & \\
\hline D3S3574 & $3 q 12$ & & & & & & \\
\hline D3S1610 & $3 q 13$ & & & & & & \\
\hline D3S1292 & $3 q 21$ & & & & & & \\
\hline D3S1272 & $3 q 27$ & & & & & & \\
\hline
\end{tabular}

Black $=$ allelic imbalance, grey $=$ informative (retention of heterozygosity), white $=$ noninformative, $\mathrm{CDR}=$ commonly deleted region. Data not shown for five additional markers that were either not informative or did not further define deleted regions in these five cases.

Table 2 Patient details of cases with structural abnormalities of chromosome 3

\begin{tabular}{|c|c|c|c|c|c|c|}
\hline$S O M$ & Sex & Treatment & Location & $\begin{array}{c}\text { Size } \\
(M D) \\
(m m)\end{array}$ & $\begin{array}{l}\text { Cell } \\
\text { type }\end{array}$ & Status \\
\hline 147 & $\mathrm{M}$ & E & $\mathrm{CB}$ & 11 & Mixed & $\begin{array}{l}\text { Alive } \\
60 \text { months }\end{array}$ \\
\hline 169 & $\mathrm{~F}$ & E & $\mathrm{CB}$ & 15 & S & $\begin{array}{l}\text { Alive } \\
60 \text { months }\end{array}$ \\
\hline 177 & M & E & $\mathrm{CB}$ & 21 & Mixed & $\begin{array}{l}\text { Dead }^{\mathrm{a}} \\
5 \text { months }\end{array}$ \\
\hline 240 & $\mathrm{~F}$ & E & C & 16 & SB & $\begin{array}{l}\text { Alive } \\
23 \text { months }\end{array}$ \\
\hline 244 & $\mathrm{~F}$ & E & $\mathrm{CB}$ & 8 & SB & $\begin{array}{l}\text { Alive } \\
23 \text { months }\end{array}$ \\
\hline
\end{tabular}

Sex: $\mathrm{M}=$ male, $\mathrm{F}=$ female. Treatment: $\mathrm{E}=$ enucleation. Location $\mathrm{C}=$ choroid, $\mathrm{CB}=$ Ciliary body. Tumour size: $\mathrm{MD}=$ maximum diameter Cell type: $\mathrm{S}=$ spindle, $\mathrm{SB}=$ spindle type $\mathrm{B}$

${ }^{a}$ Dead from metastatic disease.

complex rearrangements at 3q13-21 that were not visible by FISH or cytogenetic analysis, or by tumour heterogeneity. The translocation affecting 3q in SOM 240 was observed in approximately half of metaphases analysed, therefore tumour heterogeneity may explain why no visible abnormality was seen by cytogenetic analysis at 3p26 in SOM 240, a region exhibiting LOH in this tumour. Evidence of a structural abnormality affecting 3q was also observed in SOM 177, with allelic imbalance affecting $3 p$ and the centromeric region of $3 q$. Cytogenetic analysis of this tumour demonstrated a near- a
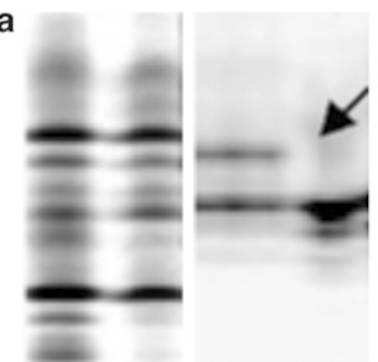

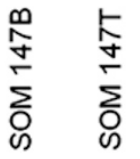

b

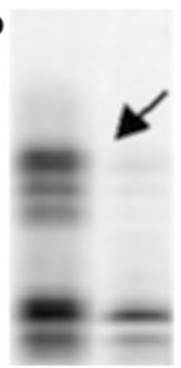

占

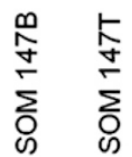

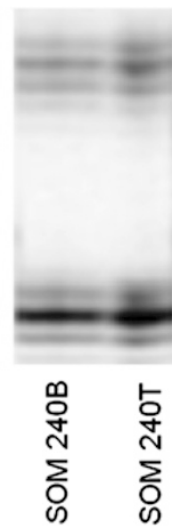

Figure 1 Analysis of microsatellites in tumours with $\mathrm{LOH}$ affecting 3p23-26. Microsatellite analysis of paired blood (B) and tumour material (T) for SOM 147 and SOM 240 at (a) D3S3525 and (b) D3S1478, confirming the presence of a breakpoint between these markers in these tumours. An arrow demonstrates $\mathrm{LOH}$.
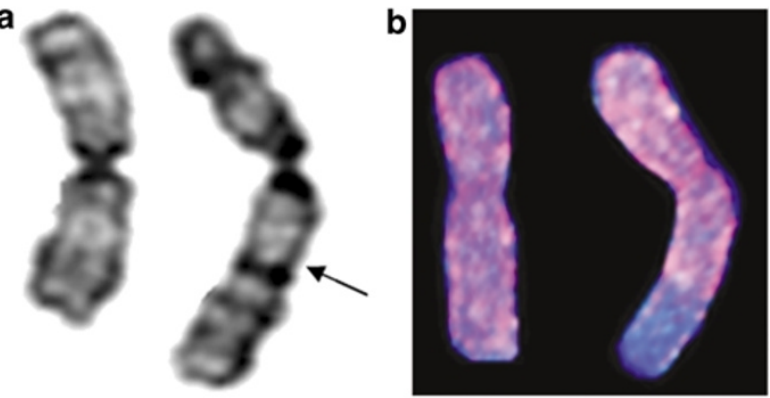

Figure 2 Chromosome 3 status of SOM 240 by cytogenetic analysis and FISH. (a) Partial karyotype of SOM 240 demonstrates the presence of one apparently normal copy of chromosome 3 and the presence of additional material at 3q23-24 (add(3) (q23-q24). (b) FISH analysis of SOM 240 using chromosome 3-specific paints, demonstrating the presence of a translocation onto chromosome $3 \mathrm{q}$.

triploid karyotype, but only two apparently normal copies of chromosome 3 . In addition, several marker chromosomes were present, potentially containing chromosome 3 material (data not shown). In the presence of two normal copies of chromosome 3 , allelic imbalance at 3 pter-3q12 and retention of heterozygosity of 3q13-3qter may actually represent a deletion of 3q13-3qter on a duplicated chromosome 3 homologue in this triploid tumour.

Two tumours demonstrated the presence of interstitial deletions around 3p11-14.2. In the first case (SOM 169) LOH was observed at D3S1481 (3p14.2), a marker within the FHIT gene, while in the second tumour (SOM 244) D3S30 (3p12) and D3S4529 (3p11) demonstrated LOH. 
Cytogenetic analysis of SOM 169 and SOM 244 revealed structural abnormalities affecting the region between 3 p14 and 3p21, and between 3p11 and 3p14, respectively, in close proximity to the regions identified by microsatellite and RFLP analysis (data not shown).

Of the 52 melanomas studied by microsatellite analysis, 24 of these were also studied by cytogenetic analysis and FISH, including four cases with structural abnormalities of chromosome 3 . Of the 20 cases with either monosomy 3 or disomy 3 , the results of molecular genetic findings correlated with cytogenetic analysis in all but a single case.

\section{Discussion}

Studies on a wide range of tumour types have indicated that recurrent loss of the entire copy of chromosome 3 is unique to uveal melanoma, in contrast to other tumour types, which preferentially lose the p-arm. ${ }^{12}$ There are several candidate TSGs on the p-arm, including Von Hippel-Lindau (VHL) at 3p25-26, Xeroderma Pigmentosum C (XPC) at 3p25, hMLH1 at 3p21.3, and fragile histidine triad (FHIT) at 3p14.2; however, to date, no TSG has been identified of the q-arm. ${ }^{12,13}$

Monosomy 3 in uveal melanomas is predominantly found in tumours that result in metastatic disease, suggesting that its occurrence results in the loss of TSGs, which, either alone or in combination with other genetic abnormalities, are prerequisites for metastatic disease. ${ }^{10,11,20}$ As the entire chromosome is lost, it seems likely that several TSGs residing on both arms are the targets, and thus in a single genetic event a reduction to homozygosity for all relevant genes would be achieved. The identification of these putative tumour suppressor loci can be directly facilitated by the study of melanomas that demonstrate partial deletions of chromosome 3.

Cytogenetic studies of uveal melanoma have identified structural abnormalities affecting chromosome 3 in a small number of cases. The presence of partial deletions of chromosome 3 at 3 p25 and at $3 q 24-26^{19}$ indicates the presence of multiple TSGs on chromosome 3. In addition to these findings, a second commonly deleted region at 3 p25 has recently been described, ${ }^{18}$ implicating a region centromeric to the region identified by Tschentscher and colleagues, suggesting the presence of more than one relevant TSG at 3p25. These findings were consistent with the notion that recurrent loss of the entire copy of chromosome 3 resulted in the disruption of one or more TSGs on each chromosomal arm.

In the present study, deletions were targeted to the 3p24-26 region, with one tumour demonstrating loss of almost the entire chromosome, retaining only markers at 3p26. Conversely, a further melanoma (SOM 240) had only loss on a marker at 3 p26, leading to the possibility of a shared deleted region in both tumours, with the smallest region implicated being 3p24-26, between D3S1293 and D3S3525. Such a region is consistent with recent findings, and would confirm this site as the possible location of a TSG of relevance to uveal melanoma. ${ }^{18,19}$ These studies expanded the number of loci investigated in the 3p25 region, and was able to eliminate the $V H L$ gene as the potential target. It is interesting that in a recent study using cDNA arrays to analyse gene expression profiles in uveal melanomas exhibiting monosomy 3 , the expression of several genes located at 3p25-26 (including fls485, a gene of unknown function and CHL1, a neuronal cell adhesion molecule) were not detectable in tumours exhibiting monosomy $3 .^{21}$ In the absence of detectable homozygous deletions, the lack of expression of CHL1 and fls485, and not 50\% reduction in expression levels, suggests that additional mechanisms other than $\mathrm{LOH}$ are involved in silencing genes on the remaining copy of chromosome 3 at sites of LOH.

A second possible region of common loss was also identified in this investigation, finding in two tumours a deletion between 3p14.2 and 3p11. Interstitial deletions affecting 3p14.2-13 have recently been reported in uveal melanomas, ${ }^{18}$ although no overlapping deletions were observed. The interstitial deletions of $3 p$ identified in this investigation were the result of chromosome abnormalities near the limit of detection by cytogenetic analysis. Armed with the results of the molecular analysis visible abnormalities of chromosome 3 were established in SOM 169 and SOM 244. Without this prior knowledge, it is likely that such deletions would be routinely missed, leading to an under representation of this region as the location of a putative TSG in uveal melanoma. The present study analysed a series of cases of uveal melanoma irrespective of whether chromosome 3 aberrations were known to be present, whereas the study by Tschentscher and associates used a series of cases with known cytogenetic abnormalities to chromosome 3. ${ }^{19}$ This could explain why deletions of 3 p12-p13 were detected only in the present study. It is noteworthy that a case of uveal melanomas has been previously described with a translocation at $3 \mathrm{p} 13$ as the sole abnormality, suggesting that a second important TSG may reside at this location. ${ }^{16}$ Furthermore, DUTT1/ ROBO1 is a putative TSG located within the deleted region identified in the present study, ${ }^{22,23}$ the expression of which is absent in uveal melanomas with monosomy $3 .^{21}$ DUTT1/ROBO1 is a neural cell adhesion molecule, as is CHL1 at 3p26, and represents a target for further study as a candidate TSG in the preferentially deleted region around 3p12-13.

Consistent with previous observations, this investigation has confirmed that loss of chromosome $3 q$ 
as well as loss of $3 p$ occurs in uveal melanoma. ${ }^{10,19,24,25}$ The present study detected two tumours (SOM 240 and SOM 177) with specific abnormalities affecting chromosome 3q. In SOM 240, a deletion affecting 3q23-24 to 3qter was observed by cytogenetic analysis. In SOM 177, allelic imbalance was observed from 3pter to 3q12; however, this tumour demonstrated a triploid karyotype with two apparently normal copies of chromosome 3 , in addition to several unidentified marker chromosomes which could potentially contain chromosome 3 material. Therefore, retention of heterozygosity at 3q13 to 3qter in this tumour may actually represent chromosome 3 duplication and subsequent deletion of 3q13 to 3qter, which would be consistent with observations of deletions affecting chromosome $3 q$ in uveal melanomas. A region of common deletion on chromosome 3 has been described in uveal melanomas at $3 q 24-26,{ }^{19}$ a site that is infrequently affected in most tumours but is deleted in osteosarcomas, paragangliomas, and pheochromocytomas. ${ }^{26-28}$ An additional tumour with a deletion involving 3q25.1-ter has also been reported in a single case of uveal melanoma, consistent with this region harbouring a TSG. ${ }^{25}$

Here we report the screening of 52 cases of uveal melanoma for partial deletions of chromosome 3, the findings of which confirm previously reported commonly deleted regions at 3p25 and 3p11-14. A highresolution follow-up study targeted to these regions is required to further localise the putative tumour suppressor loci on chromosome 3 in uveal melanoma.

\section{References}

1 Scotto J, Fraumeni JF, Lee JAH. Melanomas of the eye and other non-cutaneous sites: epidemiological aspects. J Natl Cancer Inst 1976; 56: 489-491.

2 Singh AD, Topham A. Incidence of uveal melanoma in the United States: 1973-1997. Ophthalmology 2003; 110: 956-961.

3 Gragoudas E, Egan KM, Seddon JM, Glynn RJ, Walsh SM, Finn SM et al. Survival of patients with metastases from uveal melanoma. Ophthalmology 1991; 98: 383-390.

4 Brown D, Boniuk M, Font RL. Diffuse malignant-melanoma of iris with metastases. Surv Ophthalmol 1990; 34: 357-364.

5 Sisley K, Rennie IG, Cottam DW, Potter AM, Potter CW, Rees RC. Cytogenetic findings in 6 posterior uveal melanomas - involvement of chromosome-3, chromosome-6, and chromosome-8. Genes Chromosomes Cancer 1990; 2: 205-209.

6 Prescher G, Bornfeld N, Becher R. Nonrandom chromosomal-abnormalities in primary uveal melanoma. J Natl Cancer Inst 1990; 82: 1765-1769.

7 Horsman DE, Sroka H, Rootman J, White VA. Monosomy-3 and isochromosome-8q in a uveal melanoma. Cancer Genet Cytogenet 1990; 45: 249-253.

8 Prescher G, Bornfeld N, Horsthemke B, Becher R. Chromosomal-aberrations defining uveal melanoma of poor prognosis. Lancet 1992; 339: 691-692.
9 Sisley K, Rennie IG, Parsons MA, Jacques R, Hammond DW, Bell SM et al. Abnormalities of chromosomes 3 and 8 in posterior uveal melanoma correlate with prognosis. Genes Chromosomes Cancer 1997; 19: 22-28.

10 White VA, Chambers JD, Courtright PD, Chang WY, Horsman DE. Correlation of cytogenetic abnormalities with the outcome of patients with uveal melanoma. Cancer 1998; 83: 354-359.

11 Prescher G, Bornfeld N, Hirche H, Horsthemke B, Jockel $\mathrm{KH}$, Becher R. Prognostic implications of monosomy 3 in uveal melanoma. Lancet 1996; 347: 1222-1225.

12 Knuutila S, Aalto Y, Autio K, Bjorkqvist AM, El-Rifai W, Hemmer $\mathrm{S}$ et al. DNA copy number losses in human neoplasms. Am J Pathol 1999; 155: 683-694.

13 Kok K, Naylor SL, Buys C. Deletions of the short arm of chromosome 3 in solid tumors and the search for suppressor genes. Adv Cancer Res 1997; 71: 27-92.

14 Robertson G, Herbst R, Nagane M, Huang HJ, Cavenee WK. The chromosome 10 monosomy common in human melanomas results from loss of two separate tumour suppressor loci. Cancer Res 1999; 59: 3596-3601.

15 Dahlenfors R, Tornqvist G, Wettrell K, Mark J. Cytogenetical observations in 9 ocular malignant melanomas. Anticancer Res 1993; 13: 1415-1420.

16 Blasi MA, Roccella F, Balestrazzi E, Del Porto G, De Felice $\mathrm{N}$, Roccella $\mathrm{M}$ et al. 3p13 region: a possible location of a tumor suppressor gene involved in uveal melanoma. Cancer Genet Cytogenet 1999; 108: 81-83.

17 Sisley K, Parsons MA, Garnham J, Potter AM, Curtis D, Rees RC et al. Association of specific chromosome alterations with tumour phenotype in posterior uveal melanoma. Br J Cancer 2000; 82: 330-338.

18 Parrella P, Fazio VM, Gallo AP, Sidransky D, Merbs SL. Fine mapping of chromosome 3 in uveal melanoma: Identification of a minimal region of deletion on chromosomal arm 3p25.1-p25.2. Cancer Res 2003; 63: 8507-8510.

19 Tschentscher F, Prescher G, Horseman D, White VA, Rieder $\mathrm{H}$, Anastassiou $\mathrm{G}$ et al. Partial deletions of the long and short arm of chromosome 3 point to two tumour suppressor genes in uveal melanoma. Cancer Res 2001; 61: 3439-3442.

20 Aalto Y, Eriksson L, Seregard S, Larsson O, Knuutila S. Concomitant loss of chromosome 3 and whole arm losses and gains of chromosome 1, 6 or 8 in metastasising primary uveal melanoma. Invest Ophthalmol Vis Sci 2001; 42: 313-317.

21 Tschentscher F, Husing J, Holter T, Kruse E, Dresen IG, Jockel $\mathrm{KH}$ et al. Tumor classification based on gene expression profiling shows that uveal melanomas with and without monosomy 3 represent two distinct entities. Cancer Res 2003; 63: 2578-2584.

22 Dallol A, Forgacs E, Martinez A, Sekido Y, Walker R, Kishida $\mathrm{T}$ et al. Tumour specific promoter region methylation of the human homologue of the Drosophilia Roundabout gene DUTT1 (ROBO1) in human cancers. Oncogene 2002; 21: 3020-3028.

23 Sundaresan V, Chung G, Heppell-Parton A, Xiong J, Grundy C, Roberts I et al. Homozygous deletions at $3 \mathrm{p} 12$ in breast and lung cancer. Oncogene 1998; 17: 1723-1729.

24 Naus N, van Drunen E, de Klein A, Luyten GP, Paridaens DA, Alers JC et al. Characterisation of complex chromosomal abnormalities in uveal melanoma by fluorescence in situ hybridisation, spectral karyotyping and comparative genomic hybridisation. Genes Chromosomes Cancer 2001; 30: 267-273. 
25 Scholes AGM, Liloglou T, Maloney P, Hagan S, Nunn J, Hiscott $P$ et al. Loss of heterozygosity on chromosomes 3, 9, 13 and 17, including the retinoblastoma locus, in uveal melanoma. Invest Ophthalmol Vis Sci 2001; 42: 2472-2477.

26 Kruzelock R, Murphy E, Strong L, Naylor SL, Hansen MF. Localisation of a novel tumour suppressor locus on human chromosome 3q important to osteosarcoma tumourigenesis. Cancer Res 1997; 57: 106-109.
27 Yamaguchi T, Toguchida J, Yamamuro T, Kotoura Y, Takada N, Kawaguchi $\mathrm{N}$ et al. Allelotype analysis on osteosarcomas: frequent allele loss on $3 q, 13 q, 17 p$ and 18p. Cancer Res 1992; 52: 2419-2423.

28 Edstrom E, Mahlamaki E, Nord B, Kjellman M, Karhu R, Hoog A et al. Comparative genomic hybridization reveals frequent losses of chromosomes $1 \mathrm{p}$ and $3 \mathrm{q}$ in pheochromocytomas and abdominal paragangliomas, suggesting a common genetic etiology. Am J Pathol 2000; 156: 651-659. 